\title{
The Global Stability of a Delayed Reaction-Diffusion Predator-Prey Model
}

\author{
Yu Jiang ${ }^{1, *}$, Huiming $\mathrm{Wei}^{2}$ and Xiaoli Zhang ${ }^{3}$ \\ ${ }^{1}$ Department of Public Courses, Shenzhen Institute of Information Technology, Shenzhen 518172, China \\ ${ }^{2}$ China nuclear power simulation Technology Company limited, Shenzhen 518031, China \\ ${ }^{3}$ Sino-German School Shenzhen Institute of Information Technology, Shenzhen 518172, China \\ ${ }^{*}$ Corresponding author
}

\begin{abstract}
In this article, a delayed reaction-diffusion predator-prey model with stage structure is investigated. The global stability of the coexistence states is discussed by the linearization method and the method of upper and lower solutions. Sufficient conditions of the global attractivity of nonnegative constant equilibrium of the system are obtained. Our results show that the free diffusion of the delayed reactiondiffusion system has no influence on the populations. Finally, numerical simulations are carried out to illustrate the main results.
\end{abstract}

Keywords-reaction-diffusion; time delay; the method of upper and lower solutions; global stability

\section{INTRODUCTION}

Recently many authors who are interesting in studying the dynamic behavior of the predator-prey system . In [1] used a Markovian switching process to model the telephone noise in the environment, and proposed a stochastic regime-switching predator-prey model with harvesting and distributed delays. Three species model consisting of prey were considered [2], intermediate predator that predates upon prey and top predator with intraguild predation. In this paper, we consider the system (1.1) with diffusion terms and the harvesting effort:

$$
\left\{\begin{array}{l}
\frac{\partial u_{1}}{\partial t}-D_{1} \Delta u_{1}=u_{1}(x, t)\left(a-b u_{1}(x, t)\right)-\frac{\beta u_{1}(x, t) u_{2}(x, t)}{1+c u_{1}(x, t)}, \\
\frac{\partial u_{2}}{\partial t}-D_{2} \Delta u_{2}=\gamma e^{-\omega \tau} u_{2}(x, t-\tau)-d_{3} u_{2}(x, t) \\
\quad-E u_{2}(x, t)-d_{4} u_{2}^{2}(x, t), x \in \Omega, t>0, \\
\frac{\partial u_{1}}{\partial n}=\frac{\partial u_{2}}{\partial n}=0, x \in \partial \Omega, t>0, \\
u_{1}(x, 0)=\varphi_{1}(x, 0), u_{2}(x, t)=\varphi_{2}(x, t), \quad x \in \bar{\Omega}, t \in[-\tau, 0] .
\end{array}\right.
$$

For convenience, we set:

$$
\begin{aligned}
F_{1}\left(u_{1}, u_{2}\right)=u_{1}(x, t)\left(a-b u_{1}(x, t)\right)-\frac{\beta u_{1}(x, t) u_{2}(x, t)}{1+c u_{1}(x, t)}, \\
F_{2}\left(u_{2 \tau}, u_{2}\right)=\gamma e^{-\omega \tau} u_{2}(x, t-\tau) \\
-\left(d_{3}+E\right) u_{2}(x, t)-d_{4} u_{2}^{2}(x, t),
\end{aligned}
$$

then we can rewritten the system (1.2) as the following form:

$$
\left\{\begin{array}{l}
\frac{\partial u_{1}}{\partial t}-D_{1} \Delta u_{1}=F_{1}\left(u_{1}, u_{2}\right), x \in \Omega, t>0 \\
\frac{\partial u_{2}}{\partial t}-D_{2} \Delta u_{2}=F_{2}\left(u_{2 \tau}, u_{2}\right), x \in \Omega, t>0 \\
\frac{\partial u_{1}}{\partial n}=\frac{\partial u_{2}}{\partial n}=0, x \in \partial \Omega, t>0, \\
u_{1}(x, 0)=\varphi_{1}(x, 0), u_{2}(x, t)=\varphi_{2}(x, t), x \in \bar{\Omega}, t \in[-\tau, 0] .
\end{array}\right.
$$

The system is detailed introduced in [3]. In [3], the boundedness, existence and uniqueness of the model is investigated; The existence and uniqueness of the global solution of the system are proved; The local and global stability of the constant equilibria are discussed by the linearization method and the method of upper and lower solutions, respectively. In this paper, the global stability of the coexistence states is discussed by the linearization method and the method of upper and lower solutions; Sufficient conditions of the global attractivity of nonnegative constant equilibria of the system are obtained.

The remaining parts of the paper are organized as follows. In next section, we prove the asymptotical stability of the coexistence states of the system (1.1). In section 3, we analyze the global stability of the constant equilibrium and obtain the sufficient conditions of global attractivity. Finally, we give the summary, numerical simulations and the main results of this work.

\section{Asymptotical Stability OF THE CoEXISTENCE STATES}

In this section, we assume that the system (1.4) has a pair of coexistence states $\tilde{u}_{1}(x, t), \tilde{u}_{2}(x, t)$. Then $\tilde{u}_{1}(x, t), \tilde{u}_{2}(x, t)$, satisfies the following system:

$$
\left\{\begin{array}{l}
-D_{1} \Delta u_{1}=u_{1}\left(a-b u_{1}\right)-\frac{\beta u_{1} u_{2}}{1+c u_{1}}, x \in \Omega \\
-D_{2} \Delta u_{2}=\gamma e^{-\omega \tau} u_{2}-d_{3} u_{2}-E u_{2}-2 d_{4} u_{2}^{2}, x \in \Omega \\
\frac{\partial u_{1}}{\partial n}=\frac{\partial u_{2}}{\partial n}=0, x \in \partial \Omega
\end{array}\right.
$$

We shall have the following theorem: 
Theorem 2.1. If $a d_{4}>\beta\left(\gamma e^{-\omega \tau}-d_{3}-E\right)$, then every pair of coexistence states of the system (2.1) is linearly stable as $D_{1}, D_{2} \rightarrow 0$.

Proof. Let $\left(u_{1}(x), u_{2}(x)\right)$ be a pair of coexistence states of the system (2.1). Firstly, we introduce the following eigenvalue problem:

$$
\left\{\begin{array}{l}
D_{1} \Delta \phi+\phi\left(f+u_{1} f_{u_{1}}\right)+\psi u_{1} f_{u_{2}}=\lambda \phi, x \in \Omega, \\
D_{2} \Delta \psi+\psi\left(g+u_{2} g_{u_{2}}\right)=\lambda \psi, x \in \Omega, \\
\frac{\partial \phi}{\partial n}=\frac{\partial \psi}{\partial n}=0, x \in \partial \Omega .
\end{array}\right.
$$

Since the system (2.1) is a mixed quasi-monotone system (i.e., $f_{u_{2}}<0, g_{u_{1}} \geq 0$ ), by the Krein-Rutman Theorem in [4], we know that the eigenvalue problem (2.6) in [3] has a principal eigenvalue, denote by $\lambda_{6}$, and it corresponding eigenfunction $\phi(x), \psi(x)$ can be chosen such that

$$
\phi(x)<0, \psi(x)>0, x \in \Omega
$$

It is well known that the linear stability of every pair of coexistence states of the system (2.1) is determined by the sign of $\lambda_{6}$. Therefore, we will show that $\lambda_{6}<0$. To do this, we argue by contradiction. Suppose that there exists some sequence $\left(D_{1 k}, D_{2 k}\right) \stackrel{k \rightarrow \infty}{\longrightarrow}(0,0)$ such that $\lambda_{6} \geq 0$. Let $\psi\left(x_{0}\right)=\max _{x \in \Omega} \psi(x)$, using Lemma 2.3 in [5] and (2.2)-(2.3), we get

$$
\begin{aligned}
& \max _{x \in \Omega} \psi(x)\left(-g\left(u_{1}\left(x_{0}\right), u_{2}\left(x_{0}\right)\right)\right. \\
& \left.-u_{2}\left(x_{0}\right) g_{u_{2}}\left(u_{1}\left(x_{0}\right), u_{2}\left(x_{0}\right)\right)+\lambda_{1}\right) \leq d_{4} \psi^{2}\left(x_{0}\right)
\end{aligned}
$$

and

$$
\begin{aligned}
& \max _{x \in \Omega}(-\phi(x)) \mid-f\left(u_{1}\left(x_{1}\right), u_{2}\left(x_{1}\right)\right) \\
& -u_{1}\left(x_{1}\right) f_{u_{2}}\left(u_{1}\left(x_{1}\right), u_{2}\left(x_{1}\right)\right)+\lambda_{1} \mid \leq-\frac{\beta \psi\left(x_{0}\right) u_{1}\left(x_{1}\right)}{1+c u_{1}\left(x_{1}\right)}
\end{aligned}
$$

We claim that $\lambda_{6}$ is bounded if $D_{1}, D_{2}$ are sufficiently small. In fact, suppose $\lambda_{6} \rightarrow+\infty$ as $D_{1}, D_{2} \rightarrow 0$ then, by (2.4) and (2.5), we have

$$
\max _{x \in \Omega} \psi(x) \leq \frac{d_{4} \psi^{2}\left(x_{0}\right)}{\lambda_{1}} \rightarrow 0, \text { as } D_{1}, D_{2} \rightarrow 0,
$$

and

$$
\max _{x \in \Omega}(-\phi(x)) \leq \frac{-\frac{\beta \psi\left(x_{0}\right) u_{1}\left(x_{1}\right)}{1+c u_{1}(x)}}{\lambda_{1}} \rightarrow 0 \text {, as } D_{1}, D_{2} \rightarrow 0,
$$

which contradict to the assumption $\phi<0, \psi>0$. Therefore, $\lambda_{6}$ is bounded. Define the linear operator $L: X \rightarrow Y$ given by $L\left(\begin{array}{l}\phi \\ \psi\end{array}\right)=\left(\begin{array}{l}-D_{1} \Delta \phi \\ -D_{2} \Delta \psi\end{array}\right) \quad$ where $\quad Y=[C(\Omega)]^{2} \quad$ and $X=\left\{\left(\begin{array}{c}\phi \\ \psi\end{array}\right) \in\left[C^{2}(\Omega)\right]^{2}: \frac{\partial \phi}{\partial n}=\frac{\partial \psi}{\partial n}=0\right.$, on $\left.\partial \Omega\right\}$.

Since $\lambda_{6}$ is bounded, so we can assume that $\lambda_{6} \rightarrow \bar{\lambda}$ as $D_{1}, D_{2} \rightarrow 0$.

It is well known that $L+\bar{\lambda} I: X \rightarrow Y$ is invertible and $(L+\bar{\lambda} I)^{-1}$ is compact if $\bar{\lambda}>0$ [6]. By the straight forward calculation, we have

$$
((L-A+\bar{\lambda} I) \mathbf{u}, \mathbf{u})>0 \text { for all } \mathbf{u} \neq 0
$$

where $A=\left(\begin{array}{cc}f+u_{1} f_{1} & u_{1} f_{u_{2}} \\ 0 & g+u_{2} g_{u_{2}}\end{array}\right), \mathbf{u}=(\phi(x), \psi(x))^{T}$ and $(\cdot, \cdot)$ denotes the usual $L^{2}$-inner product.

From (2.8), it follows that $L-A+\bar{\lambda} I$ is invertible. We check that

$$
(L-A+\bar{\lambda} I)^{-1}=(L+\bar{\lambda} I)^{-1}+(L-A+\bar{\lambda} I)^{-1} A(L+\bar{\lambda} I)^{-1}
$$

which implies that $(L-A+\bar{\lambda} I)^{-1}$ is compact. Next, we show that $(L-A+\bar{\lambda} I)^{-1}$ is positive. In fact, we can take $\left(\begin{array}{l}\omega \\ \xi\end{array}\right) \in Y \times Y$ with $\omega, \xi \geq 0$ and $\omega^{2}+\xi^{2} \neq 0$, such that

$$
(L-A+\bar{\lambda} I)^{-1}\left(\begin{array}{l}
\omega \\
\xi
\end{array}\right)=\left(\begin{array}{l}
\phi \\
\psi
\end{array}\right)
$$

which leads to

$$
(L-A+\bar{\lambda} I)^{-1}\left(\begin{array}{l}
\phi \\
\psi
\end{array}\right) \geq 0
$$

If $a d_{4}>\beta\left(\gamma e^{-\omega \tau}-d_{3}-E\right)$, by the strong maximum principle, we have

$$
\phi>0, \psi>0 \text { as } \phi^{2}+\psi^{2} \neq 0
$$


Combining (2.11) and (2.12) get $(L-A+\bar{\lambda} I)^{-1}$ is positive. Therefore, $(L-A+\bar{\lambda} I)^{-1}$ has a positive principle eigenvalue, denoted by $\Lambda$, i.e., there exists functions $\tilde{\phi}, \tilde{\psi}>0$ such that

$$
(L-A+\bar{\lambda} I)^{-1}\left(\begin{array}{c}
\tilde{\phi} \\
\tilde{\psi}
\end{array}\right)=\Lambda\left(\begin{array}{c}
\tilde{\phi} \\
\tilde{\psi}
\end{array}\right)
$$

which is equivalent to

$$
(L-A)\left(\begin{array}{c}
\tilde{\phi} \\
\tilde{\psi}
\end{array}\right)=\left(\frac{1}{\Lambda}-\bar{\lambda}\right) I\left(\begin{array}{c}
\tilde{\phi} \\
\tilde{\psi}
\end{array}\right)
$$

Letting $D_{1}, D_{2} \rightarrow 0$ and comparing (2.14) with (2.12), we have $-\bar{\lambda}=\frac{1}{\Lambda}-\bar{\lambda}$, which contradicts to the fact $\Lambda>0$. Therefore, $\lambda_{6}<0$ as $D_{1}, D_{2} \rightarrow 0$. This completes the proof of Theorem 2.1.

\section{Global Stability OF THE CONSTANT EQUILIBRIUM}

Denote $k_{1}\left(u_{1}, u_{2}\right)=u_{1} f\left(u_{1}, u_{2}\right), k_{2}\left(u_{1}, u_{2}\right)=u_{2} g\left(u_{1}, u_{2}\right)$. For the mixed quasi-monotone system (1.1), we give the definition of ordered upper and lower solutions.

\section{Definition}

3.1.

We $\left(\tilde{u}_{1}, \tilde{u}_{2}\right),\left(\hat{u}_{1}, \hat{u}_{2}\right) \in C(\bar{\Omega} \times[0, T]) \cap C^{2,1}(\Omega \times[0, T])$ is a pair of upper and lower solutions to the system (1.1) if

$$
\left\{\begin{array}{l}
\frac{\partial \tilde{u}_{1}}{\partial t}-D_{1} \Delta \tilde{u}_{1} \geq a \tilde{u}_{1}(x, t)-b \tilde{u}_{1}^{2}(x, t)-\frac{\beta \tilde{u}_{1}(x, t) \hat{u}_{2}(x, t)}{1+c \tilde{u}_{1}(x, t)}, \\
\frac{\partial \hat{u}_{1}}{\partial t}-D_{1} \Delta \hat{u}_{1} \leq a \hat{u}_{1}(x, t)-b \hat{u}_{1}^{2}(x, t)-\frac{\beta \hat{u}_{1}(x, t) \tilde{u}_{2}(x, t)}{1+c \hat{u}_{1}(x, t)}, \\
\frac{\partial \tilde{u}_{2}}{\partial t}-D_{2} \Delta \tilde{u}_{2} \geq \gamma e^{-\omega \tau} \tilde{u}_{2}(x, t-\tau)-\left(d_{3}+E\right) \tilde{u}_{2}(x, t)-d_{4} \tilde{u}_{2}^{2}(x, t), \\
\frac{\partial \hat{u}_{2}}{\partial t}-D_{2} \Delta \hat{u}_{2} \leq \gamma e^{-\omega \tau} \hat{u}_{2}(x, t-\tau)-\left(d_{3}+E\right) \hat{u}_{2}(x, t)-d_{4} \hat{u}_{2}^{2}(x, t), \\
\frac{\partial \hat{u}_{1}}{\partial n} \leq 0 \leq \frac{\partial \tilde{u}_{1}}{\partial n}, \frac{\partial \hat{u}_{2}}{\partial n} \leq 0 \leq \frac{\partial \tilde{u}_{2}}{\partial n}, x \in \partial \Omega, t>0, \\
\hat{u}_{1}(x, 0) \leq \varphi_{1}(x, t) \leq \tilde{u}_{1}(x, 0), x \in \bar{\Omega}, t \in[-\tau, 0], \\
\hat{u}_{2}(x, 0) \leq \varphi_{2}(x, t) \leq \tilde{u}_{2}(x, 0), x \in \bar{\Omega} .
\end{array}\right.
$$

Moreover, a pair of upper and lower solutions $\left(\tilde{u}_{1}, \tilde{u}_{2}\right),\left(\hat{u}_{1}, \hat{u}_{2}\right)$ is called ordered if $\hat{u}_{1} \leq \tilde{u}_{1}, \hat{u}_{2} \leq \tilde{u}_{2}$ in $\bar{\Omega} \times[0, T]$. Suppose that $(\tilde{c}, \hat{c})$ is a pair of ordered upper and lower solutions to the system (1.4), where $\tilde{c}_{0}=\left(\tilde{c}_{1}, \tilde{c}_{2}\right)^{T}$ and $\hat{c}=\left(\hat{c}_{1}, \hat{c}_{2}\right)^{T}$. We remark that $k_{1}\left(\tilde{u}_{1}, \tilde{u}_{2}\right)$ and $k_{2}\left(\tilde{u}_{1}, \tilde{u}_{2}\right)$ with respect to $\tilde{u}_{1}, \tilde{u}_{2}$ are continuous and mixed quasi-monotone (i.e., $\frac{\partial k_{1}}{\partial u_{2}}<0, \frac{\partial k_{2}}{\partial u_{1}}=0$ ) in $\Sigma \times \Sigma^{*}$, where $\Sigma=\left\{s \in R^{+}: \hat{c}_{1} \leq s \leq \tilde{c}_{1}\right\}, \Sigma^{*}=\left\{s \in R^{+}: \hat{c}_{2} \leq s \leq \tilde{c}_{2}\right\}$. Thus, $k_{1}\left(u_{1}, u_{2}\right)$ and $k_{2}\left(u_{1}, u_{2}\right)$ are Lipschitz continuous, i.e., for all $\left(u_{1}, u_{2}\right),\left(u_{1}^{\prime}, u_{2}^{\prime}\right) \in \Sigma \times \Sigma^{*}$, there exists $G_{i}>0(i=1,2)$ such that

$$
\left|k_{1}\left(u_{1}, u_{2}\right)-k_{1}\left(u_{1}^{\prime}, u_{2}^{\prime}\right)\right| \leq G_{1}\left(\left|u_{1}-u_{1}^{\prime}\right|+\left|u_{2}-u_{2}^{\prime}\right|\right)
$$

$$
\left|k_{2}\left(u_{1}, u_{2}\right)-k_{2}\left(u_{1}^{\prime}, u_{2}^{\prime}\right)\right| \leq G_{2}\left(\left|u_{1}-u_{1}^{\prime}\right|+\left|u_{2}-u_{2}^{\prime}\right|\right)
$$

Before using the method of upper and lower solutions in [7], we need to introduce two sequences of constant vectors $\left\{\overline{\boldsymbol{C}}^{(m)}\right\}=\left\{{\overline{C_{1}}}^{(m)}, \overline{\boldsymbol{c}}_{2}{ }^{(m)}\right\}_{m=1}^{\infty}, \quad\left\{\underline{\boldsymbol{C}}^{(m)}\right\}=\left\{\underline{c}_{1}^{(m)}, \underline{c}^{(m)}\right\}_{m=1}^{\infty}$ which satisfies

$$
\left\{\begin{array}{l}
\bar{c}_{1}^{(m)}=\bar{c}_{1}^{(m-1)}+\frac{1}{G_{1}} \bar{c}_{1}^{(m-1)}\left(a-b \bar{c}_{1}^{(m-1)}-\frac{\beta \underline{c}_{2}{ }^{(m-1)}}{1+c \bar{c}_{1}^{(m-1)}}\right), \\
{\underline{c_{1}}}^{(m)}=\underline{c}_{1}^{(m-1)}+\frac{1}{G_{1}} \underline{c}^{(m-1)}\left(a-b \underline{c}_{1}^{(m-1)}-\frac{\beta \bar{c}_{2}{ }^{(m-1)}}{1+c \underline{c}_{1}{ }^{(m-1)}}\right), \\
\bar{c}_{2}^{(m)}=\bar{c}_{2}^{(m-1)}+\frac{1}{G_{1}} \bar{c}^{(m-1)}\left(\gamma e^{-\omega \tau}-d_{3}-E-d_{4} \bar{c}_{2}^{(m-1)}\right), \\
\underline{c}^{(m)}=\underline{c}_{2}^{(m-1)}+\frac{1}{G_{1}} \underline{c}^{(m-1)}\left(\gamma e^{-\omega \tau}-d_{3}-E-d_{4} \underline{c}_{2}^{(m-1)}\right), \\
\bar{c}^{(0)}=\tilde{c}_{0}, \underline{c}^{(0)}=\hat{c}_{0}, m=1,2, \cdots
\end{array}\right.
$$

where $\left(\tilde{c}_{0}, \hat{c}_{0}\right)$ is a pair of ordered upper and lower solutions to the system (1.4). By the mixed quasi-monotone property of the system (4.1), we can prove that the following Lemma holds:

Lemma 3.1. The sequences $\left\{\bar{c}^{(m)}\right\}_{m=1}^{\infty},\left\{\underline{c}^{(m)}\right\}_{m=1}^{\infty}$ given by (3.4) satisfy

$$
\hat{c}_{0} \leq \underline{c}^{(m)} \leq \underline{c}^{(m+1)} \leq \bar{c}^{(m+1)} \leq \bar{c}^{(m)} \leq \tilde{c}_{0}, m=1,2, \cdots
$$

Lemma 3.2. Suppose that $\left(\tilde{c}_{0}, \hat{c}_{0}\right)$ is a pair of ordered upper and lower solutions to the system (1.1)and $\underline{c}, \bar{c}$ satisfy $\lim _{m \rightarrow \infty} \underline{c}^{(m)}=\underline{c}, \lim _{m \rightarrow \infty} \bar{c}^{(m)}=\bar{c}$, then the system (1.1) has a unique pair of solutions satisfying

$$
\underline{c} \leq u(x, t) \leq \bar{c} \text { as } t \rightarrow \infty
$$

for any initial functions $\hat{c}_{i} \leq \varphi_{i}(x, t) \leq \tilde{c}_{i}$ in $\bar{\Omega} \times\left[-\tau_{i}, 0\right]$ where $\tau_{1}=\tau, \tau_{2}=0$ and $i=1,2$. Moreover, if $\underline{c}=\bar{c} \triangleq c$, then there holds

$$
\lim _{t \rightarrow \infty} u(x, t)=c
$$


where $u(x, t)=\left(u_{1}(x, t), u_{2}(x, t)\right)^{T}$ and $c=\left(c_{1}, c_{2}\right)^{T}$.

Lemma 3.3. Suppose that $\left(u_{1}(x, t), u_{2}(x, t)\right)$ is a pair of global solutions to the system (1.1) and if $\varphi_{1}(x, 0) \equiv 0$ in $\bar{\Omega}$ and $\varphi_{2}(x, t) \neq 0$ in $\bar{\Omega} \times[-\tau, 0]$ then there exists $t^{*}>0$ such that

$$
u_{2}(x, t)>0 \text { in } \bar{\Omega} \times\left[t^{*},+\infty\right), \quad\left(u_{1}(x, t) \equiv 0 \text { in } \bar{\Omega} \times[0,+\infty)\right)
$$

and

$$
\left(u_{1}(x, t), u_{2}(x, t)\right) \rightarrow\left(0, \frac{\gamma e^{-\omega \tau}-d_{3}-E}{d_{4}}\right) \text { as } t \rightarrow \infty .
$$

Theorem 3.1. If $a d_{4}>\beta\left(\gamma e^{-\omega \tau}-d_{3}-E\right) \quad$ and $\gamma e^{-\omega \tau}>d_{3}+E$, then for any initial functions $\varphi_{1}(x, 0) \geq 0(\equiv 0)$ in $\bar{\Omega}$ and $\varphi_{2}(x, t) \geq 0(\not \equiv)$ in $\bar{\Omega} \times[-\tau, 0]$ the system (1.2) has a unique pair of global solutions such that

$$
\left(u_{1}(x, t), u_{2}(x, t)\right) \rightarrow\left(c_{1}^{*}, c_{2}^{*}\right) \text { as } t \rightarrow \infty
$$

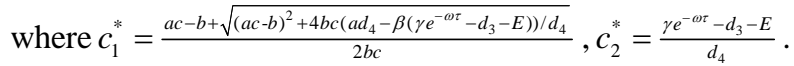

Proof. Let $W_{2}(x, t)$ be the solution of the scalar boundaryvalue problem

$$
\left\{\begin{array}{l}
\frac{\partial W_{2}}{\partial t}-D_{2} \Delta W_{2}=\gamma e^{-\omega \tau} W_{2}(x, t-\tau) \\
-\left(d_{3}+E\right) W_{2}(x, t)-d_{4} W_{2}^{2}(x, t), x \in \bar{\Omega}, t>0, \\
\frac{\partial W_{2}}{\partial n}=0, x \in \partial \bar{\Omega}, t>0, \\
W_{2}(x, t)=\varphi_{2}(x, t) \geq 0, x \in \bar{\Omega}, t \in[-\tau, 0] .
\end{array}\right.
$$

Applying Lemma 2.1 in [3], we have

$$
u_{2}(x, t) \leq W_{2}(x, t),(x, t) \in \bar{\Omega} \times[0,+\infty)
$$

By Lemma 3.1 and Lemma 3.3, it follows that

$$
W_{2}(x, t) \rightarrow \frac{\gamma e^{-\theta t}-d_{3}-E}{d_{4}}, t \rightarrow \infty
$$

Hence, for any $\varepsilon>0$, there always exists $t^{* *}>0$ as $t>t^{* *}$ such that

$$
u_{2}(x, t) \leq W_{2}(x, t) \leq \frac{\gamma e^{-\omega \tau}-d_{3}-E}{d_{4}}+\varepsilon
$$

Let $W_{1}(x, t)$ be the solution of the scalar boundary-value problem

$$
\begin{aligned}
& \frac{\partial W_{1}}{\partial t}-D_{1} \Delta W_{1} \leq W_{1}(x, t)\left(a-b W_{1}(x, t)\right. \\
& \left.-\frac{\beta\left(\gamma e^{-\omega \tau}-d_{3}-E+d_{4} \varepsilon\right)}{d_{4}\left(1+\frac{a c}{b}\right)}\right),(x, t) \in \bar{\Omega} \times\left[t^{* *},+\infty\right)
\end{aligned}
$$

In fact, $a-b W_{1}(x, t)>0, W_{1}(x, t)=\varphi_{1}\left(x, t^{* * *}\right) \geq 0, x \in \bar{\Omega}$.

By the quasi-monotone property of $k_{1}\left(u_{1}, u_{2}\right)$, we obtain

$$
u_{1}(x, t) \leq W_{2}(x, t),(x, t) \in \bar{\Omega} \times\left[t^{* *},+\infty\right)
$$

Now we consider the scalar boundary-value problem

$$
\left\{\begin{array}{l}
\frac{\partial W_{1}}{\partial t}-D_{2} \Delta W_{1}=W_{1}(x, t)\left(a-b W_{1}(x, t)\right. \\
\left.-\frac{\beta\left(\gamma e^{-\omega \tau}-d_{3}-E+d_{4} \varepsilon\right)}{d_{4}\left(1+\frac{a c}{b}\right)}\right),(x, t) \in \bar{\Omega} \times\left[t^{* *},+\infty\right), \\
\frac{\partial W_{1}}{\partial n}=0,(x, t) \in \partial \bar{\Omega} \times\left[t^{* *},+\infty\right), \\
W_{1}(x, t)=\varphi_{1}\left(x, t^{* *}\right) \geq 0, x \in \bar{\Omega} .
\end{array}\right.
$$

By [8], we have

$$
W_{1}(x, t) \rightarrow \frac{a d_{4}}{b}\left(1+\frac{a c}{b}\right)-\frac{\beta}{b}\left(\gamma e^{-\omega \tau}-d_{3}-E+d_{4} \varepsilon\right) \text { as } t
$$

sufficiently large. Therefore, for the above given $\varepsilon$, there exists $t_{0}>0$ satisfying

$$
\begin{aligned}
& u_{1}(x, t) \leq \frac{a d_{4}}{b}\left(1+\frac{a c}{b}\right)-\frac{\beta}{b}\left(\gamma e^{-\omega \tau}-d_{3}\right. \\
& \left.-E+d_{4} \varepsilon\right),(x, t) \in \bar{\Omega} \times\left[t_{0},+\infty\right)
\end{aligned}
$$

Let

$$
\begin{cases}\tilde{c}_{1}=\frac{a d_{4}}{b}\left(1+\frac{a c}{b}\right)-\frac{\beta}{b}\left(\gamma e^{-\omega \tau}-d_{3}-E+d_{4} \varepsilon\right), \hat{c}_{1}=\delta_{1}, \\ \tilde{c}_{2}=\frac{\gamma e^{-\omega \tau}-d_{3}-E}{d_{4}}+\varepsilon, & \hat{c}_{2}=\delta_{2},\end{cases}
$$

where $\varepsilon, \delta_{1}$ and $\delta_{2}$ are sufficiently small positive constants. By $a d_{4}>\beta\left(\gamma e^{-\omega \tau}-d_{3}-E\right)$ and $\gamma e^{-\omega \tau}>d_{3}+E$, applying Lemma 3.1 in [9] and Lemma 2.1 in [3], for $t$ sufficiently large, we obtain that 


$$
\hat{c}_{1} \leq u_{1}(x, t) \leq \tilde{c}_{1}, \hat{c}_{2} \leq u_{2}(x, t) \leq \tilde{c}_{2},
$$

$$
\begin{gathered}
\tilde{c}_{1}=\frac{a d_{4}}{b}\left(1+\frac{a c}{b}\right)-\frac{\beta}{b}\left(\gamma e^{-\omega \tau}-d_{3}-E+d_{4} \varepsilon\right) \Rightarrow a-b \tilde{c}_{1}-\frac{\beta \tilde{c}_{2}}{1+c \tilde{c}_{1}} \leq 0 ; \\
\tilde{c}_{2}=\frac{\gamma e^{-\omega \tau}-d_{3}-E}{d_{4}}+\varepsilon \Rightarrow \frac{\gamma e^{-\omega \tau}-d_{3}-E}{d_{4}} \tilde{c}_{2}-\tilde{c}_{2}^{2} \leq 0 .
\end{gathered}
$$

So, it is not difficult to prove that $\hat{c}_{1}=\delta_{1}$ and $\hat{c}_{2}=\delta_{2}$ satisfy (3.1). Hence, $\left(\tilde{c}_{1}, \tilde{c}_{2}\right),\left(\hat{c}_{1}, \hat{c}_{2}\right)$ are a pair of coupled upper and lower solutions of the system (1.4).

By Lemma 3.2, we obtain that $\underline{c}$ and $\bar{c}$ satisfy (3.7). Since $a d_{4}>\beta\left(\gamma e^{-\omega \tau}-d_{3}-E\right)$ and $\gamma e^{-\omega \tau}>d_{3}+E$, and the following equations have unique positive solutions:

$$
c_{1}\left(a-b c_{1}-\frac{\beta c_{2}}{1+c c_{1}}\right)=0, c_{2}\left(\gamma e^{-\omega \tau}-d_{3}-E+d_{4} c_{2}\right)=0
$$

Therefore,

$$
\bar{c}_{1}=\underline{c}_{1}=c_{1}^{*}, \bar{c}_{2}=\underline{c}_{2}=c_{2}^{*} .
$$

So $\lim _{t \rightarrow \infty} u_{1}(x, t)=c_{1}^{*}, \lim _{t \rightarrow \infty} u_{2}(x, t)=c_{2}^{*}$. This completes the proof of Theorem 3.1.

Observing by Theorems 3.1, 3.2 in [3] and Theorems 2.1, 3.1, we get some results as follows:

Theorem 3.2. If $a d_{4}>\beta\left(\gamma e^{-\omega \tau}-d_{3}-E\right)$ and $\gamma e^{-\omega \tau}>d_{3}+E$, then the positive equilibrium $E_{4}\left(c_{1}^{*}, C_{2}^{*}\right)$ is globally asymptotically stable as $D_{1}, D_{2}$ are sufficiently small.

Theorem 3.3. If $a d_{4}>\beta\left(\gamma e^{-\omega \tau}-d_{3}-E\right) \quad$ and $\gamma e^{-\omega \tau}>d_{3}+E$, then the positive equilibrium $E_{4}\left(c_{1}^{*}, c_{2}^{*}\right)$ is globally asymptotically stable.

\section{SUMMARY}

In this work, a delayed reaction-diffusion predator-prey model with stage structure and continuous harvesting for predator is discussed. By using the linearization method and the method of upper and lower solutions, the global stability of the coexistence states of the system is investigated. Sufficient conditions of the global attractivity of nonnegative constant equilibria of the system are obtained. By Theorem 2.1 and 3.1, one can see that every pair of coexistence states of the system
(2.1) is linearly stable as $D_{1}, D_{2} \rightarrow 0$ and $a d_{4}>\beta\left(\gamma e^{-\omega \tau}-d_{3}-E\right)$. By Theorem 3.2, if $a d_{4}>\beta\left(\gamma e^{-\omega \tau}-d_{3}-E\right)$ and $\gamma e^{-\omega \tau}>d_{3}+E$, then the positive equilibrium $E_{4}\left(c_{1}^{*}, c_{2}^{*}\right)$ is globally asymptotically stable as $D_{1}, D_{2}$ are sufficiently small. By Theorem 3.3, one can see that the positive equilibrium $E_{4}\left(c_{1}^{*}, C_{2}^{*}\right)$ is globally asymptotically stable if the following conditions holds: $a d_{4}>\beta\left(\gamma e^{-\omega \tau}-d_{3}-E\right)$ and $\gamma e^{-\omega \tau}>d_{3}+E$. Finally, numerical simulations are carried out to illustrate the main results. Our results show that the free diffusion of the delayed reaction-diffusion system has no influence on the populations.

In the following, we give some examples to illustrate our main results on the global convergence of positive solutions of

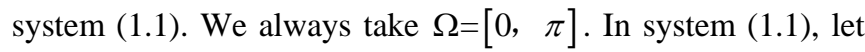
$D_{1}=2, D_{2}=0.5, \mathrm{a}=2, \mathrm{~b}=4.5, \mathrm{c}=2, \beta=4, \gamma=0.5, \Omega=0.5, \tau=1$, $d_{3}=0.1, d_{4}=2, E=0.1$. In this example, we take $D_{1}=2, D_{2}=0.5$, and they are small. Then it is easy to show that system (1.1) has a unique positive equilibrium $\quad E_{4}(0.3808,0.1262) \quad$ C Clearly, $a d_{4}>\beta\left(\gamma e^{-\omega \tau}-d_{3}-E\right)$. By Theorem 3.1, one can see that the positive solution $\left(u_{1}(x, t), u_{2}(x, t)\right)$ of system (1.1) converges to the positive equilibrium $E_{4}(0.3808,0.1262)$ (see Figure. 1). In system (1.1), let $D_{1}=0.001, D_{2}=0.002, a=2, b=4.5, c=2$,

$\beta=4, \gamma=0.5, \Omega=0.1, \tau=1, d_{3}=0.1, d_{4}=2, E=0.1 . \quad$ In $\quad$ this example, we take $D_{1}=0.001, D_{2}=0.002$, and they are very small. Then it is easy to show that system (1.1) has a unique positive equilibrium $E_{4}(0.3808,0.1262)$ Clearly, $a d_{4}>\beta\left(\gamma e^{-\omega \tau}-d_{3}-E\right)$. By Theorem 2.1 and 3.2, one can see that the positive solution $\left(u_{1}(x, t), u_{2}(x, t)\right)$ of system (1.1) converges to the positive equilibrium $E_{4}(0.3808,0.1262)$. (see Figure. 2). In system (1.1), let $D_{1}=10, \quad D_{2}=30, \quad \mathrm{a}=2, \quad \mathrm{~b}=4.5, \quad \mathrm{c}=2$, $\beta=4, \gamma=0.5, \Omega=0.1, \tau=1, d_{3}=0.1, d_{4}=2, E=0.1 . \quad$ In $\quad$ this example, we take $D_{1}=10, D_{2}=30$, and they are bigger. Then it is easy to show that system (1.1) has a unique positive equilibrium $\quad E_{4}(0.3808,0.1262)$ Clearly, $a d_{4}>\beta\left(\gamma e^{-\omega \tau}-d_{3}-E\right)$. By Theorem 3.3, one can see that the positive solution $\left(u_{1}(x, t), u_{2}(x, t)\right)$ of system (1.1) converges to the positive equilibrium $E_{4}(0.3808,0.1262)$ (see Figure. 3). 

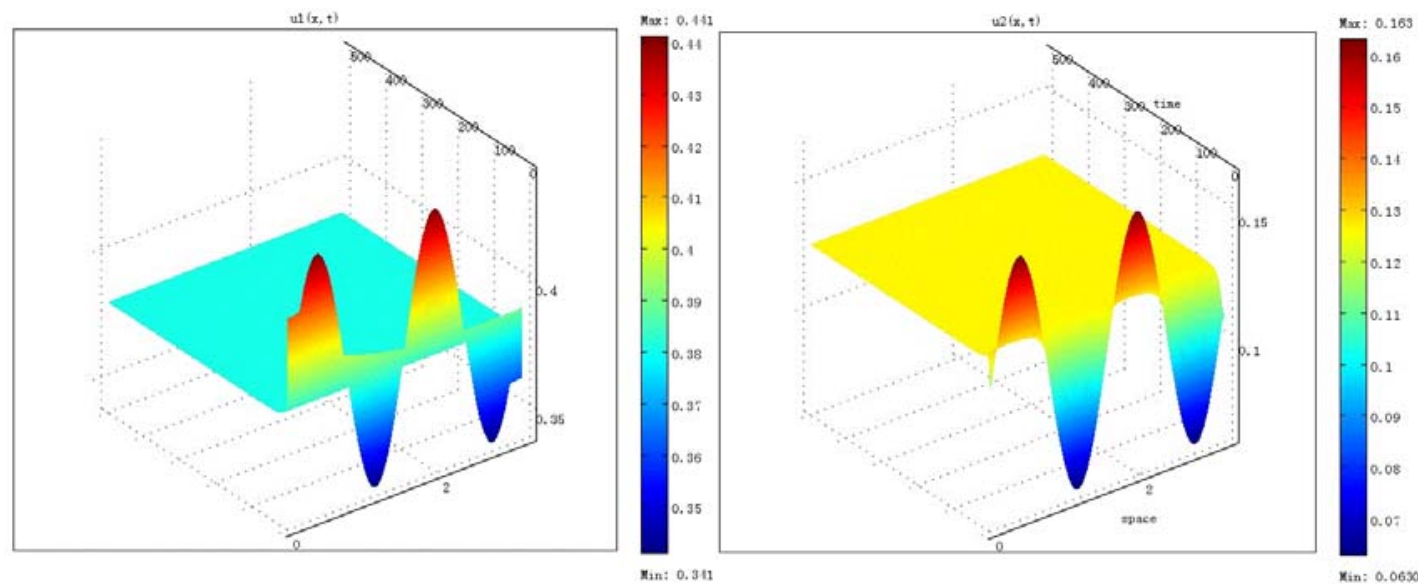

FIGURE I. THE TEMPORAL SOLUTION FOUND BY NUMERICAL INTEGRATION OF SYSTEM (1.2) WITH $D_{1}=2, D_{2}=0.5, \mathrm{a}=2, \mathrm{~b}=4.5, \mathrm{c}=2, \beta=4, \gamma=0.5, \Omega=0.1, \tau=1, d_{3}=0.1, d_{4}=2, E=0.1$.
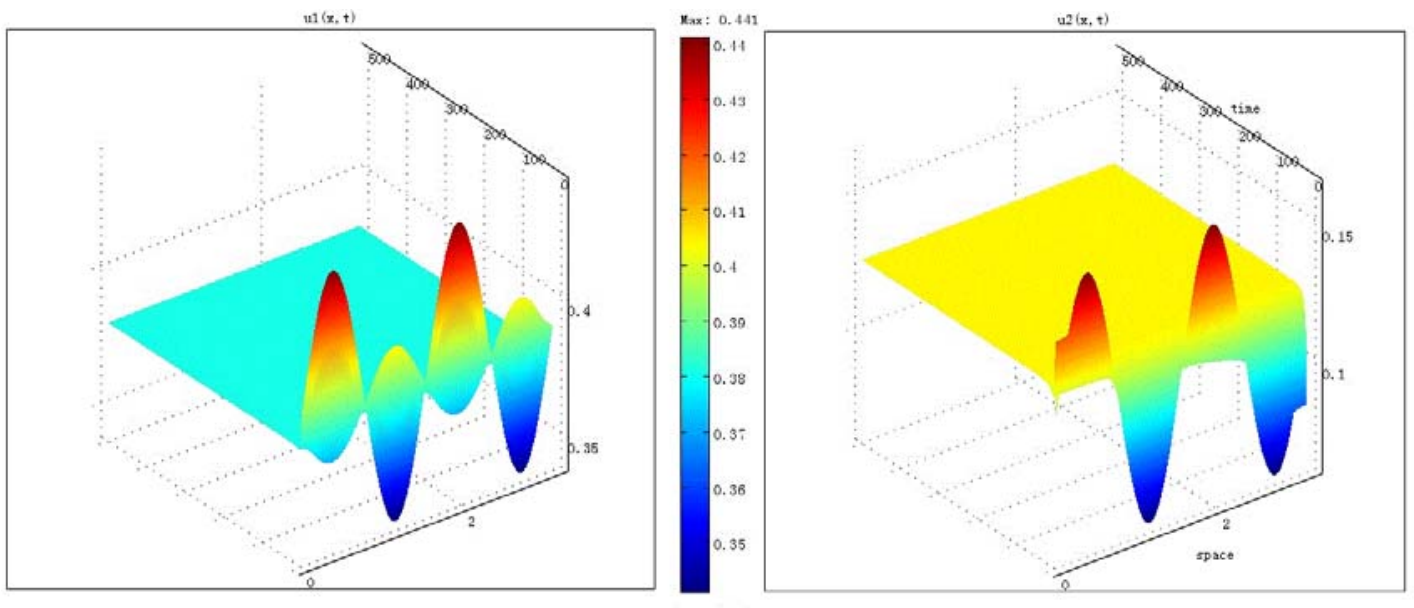

Yin: 0.341

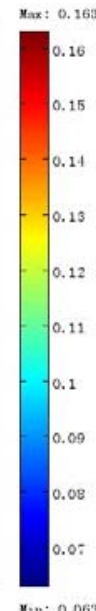

FIGURE II. THE TEMPORAL SOLUTION FOUND BY NUMERICAL INTEGRATION OF SYSTEM (1.2) WITH $D_{1}=0.001, D_{2}=0.002, \mathrm{a}=2, \mathrm{~b}=4.5, \mathrm{c}=2, \beta=4, \gamma=0.5, \Omega=0.1, \tau=1, d_{3}=0.1, d_{4}=2, E=0.1$.
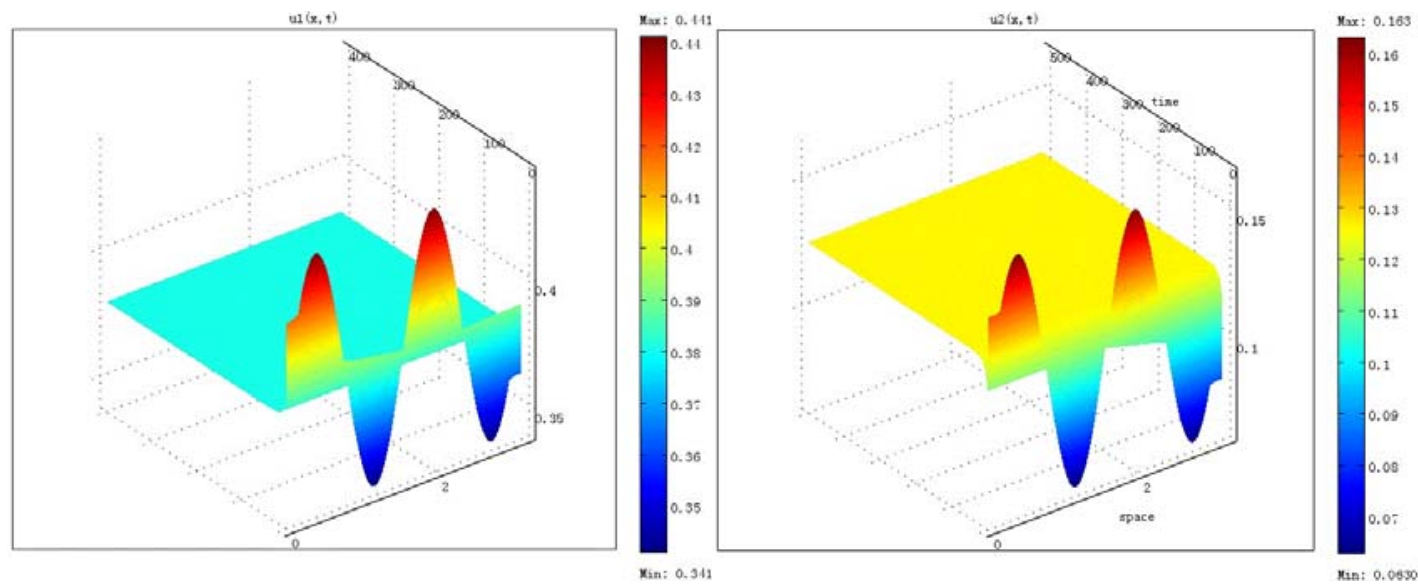

FIGURE III. THE TEMPORAL SOLUTION FOUND BY NUMERICAL INTEGRATION OF SYSTEM (1.2) WITH $D_{1}=10, D_{2}=30, \mathrm{a}=2, \mathrm{~b}=4.5, \mathrm{c}=2, \beta=4, \gamma=0.5, \Omega=0.1, \tau=1, d_{3}=0.1, d_{4}=2, E=0.1$. 


\section{ACKNOWLEDGMENT}

The research was financially supported by Finite Element Method for differential equation (11400-17-020203-0639).

\section{REFERENCES}

[1] M. Liu, X. He, and J. Yu, "Dynamics of a stochastic regime-switching predator-prey model with harvesting and distributed delays," Nonlinear Analysis: Hybrid Systems, Vol. 28, pp. 87-104, May 2018.

[2] D. Sen, S. Ghorai, and M. Banerjee, "Complex dynamics of a three species prey-predator model with intraguild predation," Ecological Complexity, Vol. 34, pp. 9-22, May 2018.

[3] Y. Jiang, and H. Wei, "The Boundedness, Existence, Uniqueness and Stability of a Delayed Reaction-Diffusion Predator-Prey Model,” 2017 International Conference on Mathematics, Modelling and Simulation Technologies and Applications (MMSTA 2017), ISBN: 978-1-60595530-8, Part 1, pp. 24-31, December 24-25, 2017, Xiamen, China.

[4] M.G. Krein, and M.A. Rutman, "Linear operators leaving invariant a cone in a Banach space,” Amer. Math. Soc. Transl. Ser., Vol. 226, pp. 1128, 1950.

[5] Z.H. Ge, and Y.N. He, "Diffusion effect and stability analysis of a predator-prey system described by a delayed reaction-diffusion equations,” J. Math. Anal. Appl., Vol. 339, pp. 1432-1450, 2008.

[6] L.C. Evans, Partial Differential Equations, American Mathematical Society, Providence, RI, 1998.

[7] C.V. Pao, Nonlinear Parabolic and Elliptic Equations, Plenum Press, New York, 1992.

[8] M. Wang, Nonlinear Partial Differential Equations of Parabolic Type, Science Press, Beijing, 1993.

[9] C.V. Pao, "Dynamics of nonlinear parabolic systems with time delays," J. Math. Anal. Appl., Vol. 198, pp. 751-779, 1996. 